\title{
Presurgical Mapping of Brain Tumors Using Statistical Probabilistic Anatomical Maps
}

\author{
Jin Su Kim1,2,3*, Gi Jeong Cheon", Sang Moo Lim ${ }^{1}$ \\ ${ }^{1}$ Molecular Imaging Research Center, Korea Institute of Radiological and Medical Sciences, Seoul, Korea \\ ${ }^{2}$ Korea Drug Development Platform Using Radio-Isotope (KDePRI), Seoul, Korea \\ ${ }^{3}$ Radiologcial and Medico-Oncological Sciences, University of Science and Technology (UST), Seoul, Korea \\ ${ }^{4}$ Department of Nuclear Medicine, Seoul National University College of Medicine, Seoul, Korea \\ Email: ${ }^{*}$ kjs@kirams.re.kr
}

Received 21 August 2015; accepted 21 September 2015; published 24 September 2015

Copyright (C) 2015 by authors and Scientific Research Publishing Inc.

This work is licensed under the Creative Commons Attribution International License (CC BY).

http://creativecommons.org/licenses/by/4.0/

(c) (i) Open Access

\section{Abstract}

Purpose: Multi-tracer neuroimaging is widely used for the diagnosis, radiotherapy, and neurosurgery of brain gliomas. Anatomical and functional information is important to avoid having neurological deficits induced during the resection or radiation therapy of brain gliomas. The aim of this study was to investigate presurgical anatomical labeling of brain gliomas on FLT and FET using statistical probabilistic anatomic maps (SPAM), which are images of cerebral cortical, cerebellar, and subcortical volumes of interest (VOIs). Methods: FDG, FLT, and FET PET scans were acquired. FLT and FET PET images were coregistered to the FDG PET images, which were then spatially normalized onto the target brain. An inverse spatial normalization parameter was calculated and applied to SPAM. For the anatomical labeling of brain glioma regions, the volumes of brain gliomason FLT and FET images were extracted using segmentation. Probabilistic information of the glioma region was then calculated using SPAM and the segmented glioma volumes. SPM and an in-house program were used for image processing. Results: The probability of SPAM labeling a brain glioma region could be extracted using the inverse normalized SPAM and segmented glioma regions. In a sample case, the probabilistic anatomical region of the glioma included $21 \%$ of the postcentral gyrus, $12 \%$ of the superior parietal gyrus, and $6 \%$ of the angular gyrus. Conclusion: Anatomical information about brain gliomas could be extracted using SPAM. This proposed method would be optional for presurgical mapping, to avoid an additional functional mapping study that might otherwise be necessary to avoid producing neurological deficits.

\section{Keywords}

SPAM, Presurgical Mapping, Brain Tumor

\footnotetext{
${ }^{*}$ Corresponding author.
}

How to cite this paper: Kim, J.S., Cheon, G.J. and Lim, S.M. (2015) Presurgical Mapping of Brain Tumors Using Statistical Probabilistic Anatomical Maps. J. Biomedical Science and Engineering, 8, 653-658. 


\section{Introduction}

Brain tumors are the most common solid tumors, and are typically evaluated by ${ }^{18} \mathrm{~F}$ FDG PET (FDG) or by combined FDG and MR imaging [1]. The hybrid PET/MR allows for molecular, anatomical, and functional imaging such as ASL, proton-spectroscopy, ${ }^{11} \mathrm{C}$ methionine PET, and ${ }^{68} \mathrm{Ga}$ DOTATOC PET [2]. 3'-deoxy-3'-[ $\left.{ }^{18} \mathrm{~F}\right]$ fluorothymidine (FLT) PET has been found useful for the noninvasive grading of gliomas, their prognostic assessment, and evaluation of response to malignant glioma treatments [3]. O- $\left(2-\left[{ }^{18} \mathrm{~F}\right]\right.$ fluoroethyl)-L-tyrosine (FET) has gained ground for the diagnostic work-up of brain tumors, because it overcomes the limitations of FDG PET by providing a much higher tumor to non-tumor contrast [4].

Presurgical evaluation of language in brain tumor patients is important. For example, functional magnetic resonance imaging is used to assess laterality in preoperative brain tumor cases [5]-[7]. In addition, navigated transcranial magnetic stimulation (nTMS) is increasingly used in presurgical brain mapping. Preoperative nTMS results correlate well with direct cortical stimulation (DCS) data in the identification of the primary motor cortex [8].

A statistical probabilistic anatomic map (SPAM) is an atlas-based volume of interest (VOI) that is used to obtain the regional counts from spatially normalized individual images into standardized brain templates. For example, the SPAM images of the Montreal Neurological Institute, consisting of 98 VOIs, were used to objectively calculate the PET or SPECT counts in neocortical, cerebellar, and subcortical VOIs. Each image consisted of probabilities belonging to specific regions. The SPAM probability-weighted mean counts of frontal cortex, temporal cortex, parietal cortex, occipital cortex, whole cerebral cortex, cerebellar cortex, basal ganglia, and thalamus for each hemisphere were obtained from the 98VOIs using the probabilistic brain atlas. Counts were calculated by dividing the probability-weighted sum of all voxels in the VOI by the sum of the probability sum of the VOI and the number of voxels [9] [10]. SPAM was used to quantify brain perfusion SPECT [10], surgical prognosis of temporal lobe epilepsy [11], and perfusion and glucose metabolism of epileptogenic zones in temporal lobe epilepsy [12].

In this present study, we developed a SPAM-assisted presurgical evaluation of brain tumors using the inverse spatial normalization mapping technique provided in the SPM software's toolbox. Our method as developed herein could provide functional mapping without the need for any additional data acquisition by imaging techniques such as fMRI, nTMS, or DCS.

\section{Material and Methods}

\subsection{Patients}

The method developed for this study was retrospectively applied to already acquired PET data in our institute [3]. The patients who were previously diagnosed with glioma and who were treated with multiple therapies (such as operation, chemotherapy and radiotherapy) within a 7-year period from 2003 to 2009. Our previous study was approved by the institutional review board of the Korean Cancer Center Hospital, and written informed consent was obtained from patients. Patients (13 men and 7 women, age range: 31 - 72 years, mean age: $51.7 \pm 12.6$ years) with newly diagnosed gliomas who underwent MRI, FLT, and FET PET were included in our study. All patients had been referred to our institute for initial diagnosis. A pathological diagnosis was obtained by stereotactic biopsy or open surgery. Grading of the tumor was performed according to the World Health Organization (WHO) grading system for gliomas. Three patients had grade II, two had grade III, and fifteen had grade IV gliomas.

\subsection{PET Study}

Briefly, the PET acquisition process was described as follows: Twenty patients underwent FDG, FLT, and FET PET within 3 days. The images of the brain were obtained thirty minutes after intravenous injection of approximately 370 MBq of FDG, FLT, or FET. An ECAT HR+ PET scanner (Siemens Medical System, Knoxville, TN) was used. A 20-min emission scan $(128 \times 128$ matrix $)$ and a 10-min post-emission transmission scan using a

${ }^{68} \mathrm{Ge}$ source were performed. The attenuation-corrected images were reconstructed with an ordered subset expectation maximization (OSEM) algorithm (2 iterations and 16 subsets) with segmented attenuation correction.

\subsection{Coregistration}

In this study, performance of 4 registration methods including mutual information (MI), normalized mutual 
information (NMI), normalized cross correlation (NCC), and entropy correlation coefficient (ECC) were compared. For the implementation of image registration, file format was converted into analyze format. Individual images were registered using 4 registration methods including MI, NMI, NCC and ECC. Image registration was performed using SPM5. To assess the correctness of the image registration procedure, we used image fusion software such as MRIcro in which the fused image of the reference image and source image can be visualized with various label of the opaqueness.

\subsection{Glioma Region Mapping Using Statistical Probabilistic Anatomic Map}

In this study, we used the Korean version of SPAM, which was developed by the Seoul National University group [13]. SPAM images of the Seoul National University consisting of 152 VOIs were used to objectively calculate the PET counts in the neocortical, cerebellar, and subcortical VOIs. Each image consisted of the probabilities that belonged to its specific regions.

Individual FET PET or FLT PET data was coregistered to FDG PET data using the mutual information method provided in the SPM 8 software. The FDG PET image was then spatially normalized onto Korean standard templates [13] by both linear and nonlinear transformations. The inverse spatial normalization parameter was calculated and applied to SPAM. For the anatomical labeling of brain glioma regions, the volume of brain gliomas on FLT and FET images was extracted using segmentation. The probabilistic information of the glioma region was then calculated using SPAM and the segmented glioma volume.

\section{Results}

Figure 1 shows the result of co-registration. Image registration of FDG \& FLT, FDG \& FET, and FDG \& MR was successfully performed using MI, NMI, and ECC methods. However, image registration of FDG \& FLT and FDG \& MR could not be performed using NCC method. Yellow arrow indicates the mismatch of coregistration.

Figure 2(a) shows the individual FDG PET, FLT PET, and FET PET scans. Figure 2(b) demonstrates the

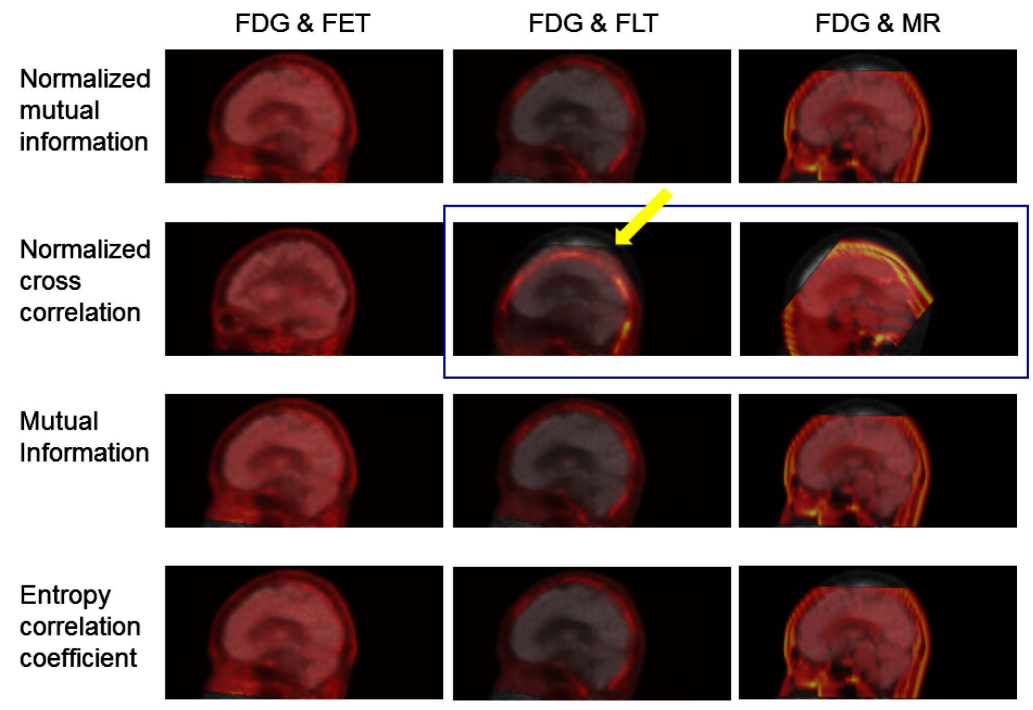

Figure 1. Performance of 4 registration methods including mutual information (MI), normalized mutual information (NMI), normalized cross correlation (NCC), and entropy correlation coefficient (ECC) were compared. Individual images were registered using 4 registration methods including MI, NMI, NCC and ECC. To assess the correctness of the image registration procedure, we used image fusion software such as MRIcro in which the fused image of the reference image and source image can be visualized with various label of the opaqueness. Image registration of FDG \& FLT, FDG \& FET, and FDG \& MR was successfully performed using MI, NMI, and ECC methods. However, image registration of FDG \& FLT and FDG \& MR could not be performed using NCC method. 
coregistration of FDG PET and FET PET, and of FDG PET and FLT PET data.

Figure 3 shows the preprocessing step for anatomical mapping using SPAM. The FDG PET image was spatially normalized onto the target brain and the inverse spatial normalization parameter was calculated. Inverse spatial normalization was applied to SPAM onto coregistered FDG PET, FLT PET, and FET PET scans.

Figure 4 shows the application of inverse normalized SPAM in regions such as the postcentral gyrus, precentral gyrus, and superior parietal gyrus onto FLT PET imaging data. The probability of a SPAM-labeled brain glioma region could be extracted using inverse normalized SPAM and the segmented glioma region. In the sample case, the probabilistic anatomical region of the glioma included $8.9 \%$ of the precentral gyrus, 36.8\% of the postcentral gyrus, $21.7 \%$ of the superior parietal gyrus, $10.9 \%$ of the supramarginal gyrus, $6.6 \%$ of the angular gyrus, and $14.8 \%$ of Brodmann's area 44.

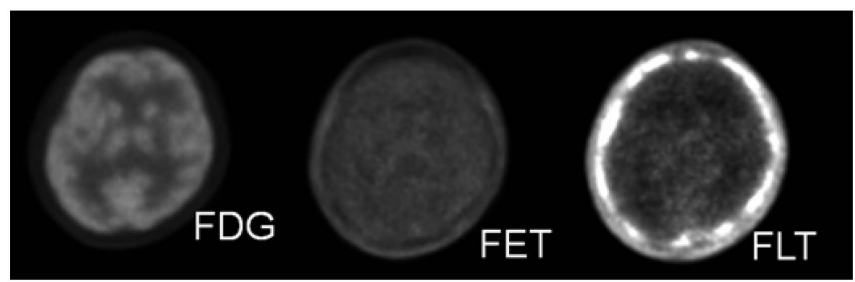

(a)

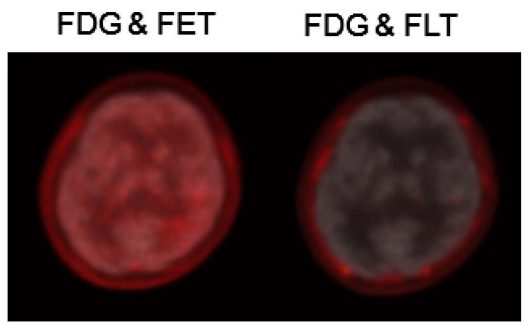

(b)

Figure 2. (a) Individual FDG PET, FLT PET, and FET PET scans; (b) Coregistered FDG PET and FET PET, and FDG PET and FLT PET, imaging data.

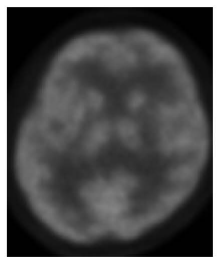

Individual data

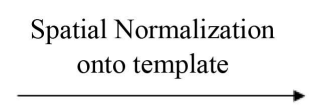

onto template

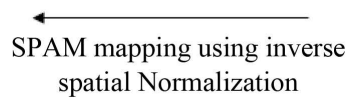

spatial Normalization

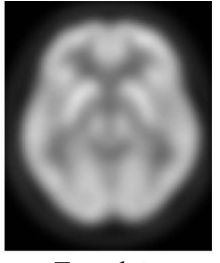

Template

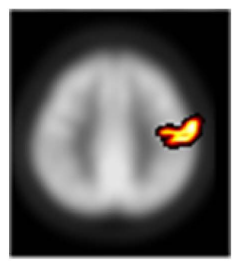

SPAM on template

Figure 3. The preprocessing step for anatomical mapping using a statistical probabilistic anatomic map (SPAM). An FDG PET image was spatially normalized onto the target brain and the inverse spatial normalization parameter was calculated. Inverse spatial normalization was applied using SPAM onto coregistered FDG PET, FLT PET, and FET PET scans. 


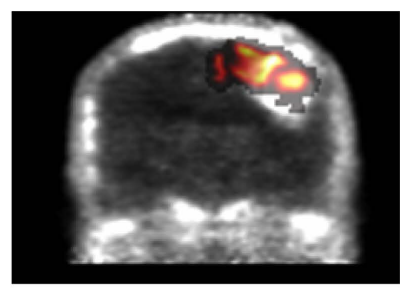

Postcentral gyrus

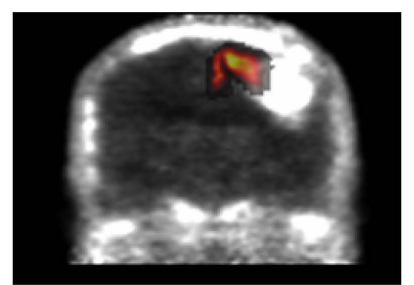

Precentral gyrus

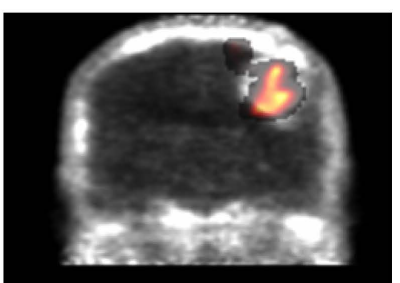

Superior parietal gyrus

Figure 4. Application of inverse normalized SPAM of regions including the postcentral gyrus, precentral gyrus, and superior parietal gyrus, onto ${ }^{18} \mathrm{~F}$ FLT PET imaging data. The probability of a SPAM-labeled brain glioma region could be extracted using a combination of the inverse normalized SPAM and the segmented glioma region.

\section{Discussion}

In our present study, we developed a SPAM-assisted presurgical evaluation of brain tumors using an image processing step involving SPM, but without any additional presurgical data acquisition. For the successful implementation of inverse spatial normalization, spatial normalization onto the template is important [14]. In our present study, we applied the SPAM-assisted presurgical evaluation of brain tumors to FLT PET data because FLT PET data lack specific anatomical information. Due to high contrast in the tumor to non-tumor ratio, we used FDG PET for spatial normalization. The FLT image was coregistered to an FDG PET image. In our previous study, we compared 4 different coregistration methods: Mutual information, normalized mutual information, normalized cross correlation, and entropy correlation coefficient. We found that image registration of FDG and FLT, FDG and FET, and FDG and MR was successfully performed using mutual information, normalized mutual information, and entropy correlation coefficient methods, but not via the normalized cross correlation method [15]. We found that inverse spatial normalization of SPAM onto coregistered FLT PET information could provide enhanced anatomical information on the native spatial space of FLT PET data.

Many studies on the feasibility of fMRI in presurgical mapping for brain tumors have been introduced [5]-[7] [16]. Because the fMRI study of cancer patients is difficult, SPAM-assisted presurgical mapping for brain tumor patients would be a more feasible option for clinics.

Presurgical mapping is also important for epilepsy surgery evaluations in which the epileptic focus is to be located. Typically, the presurgical evaluation was performed using MRI, SPECT, PET, fMRI, or MEG [17]-[19]. Although developed in brain glioma cases, our SPAM-assisted presurgical mapping technique could apply to presurgical mapping for epilepsy surgery as well.

Taken together, the data in the present study demonstrate the feasibility of SPAM-assisted presurgical mapping for brain tumors. However, because there would be individual differences in brain function, and anatomical changes due to the tumor's volume, our SPAM-assisted mapping method could best be used as an instant mapping method just prior to an fMRI study, or in cases when presurgical mapping using fMRI or other methods is not available.

Our proposed SPAM-assisted presurgical mapping could provide efficient tool for avoiding of producing neurological deficits without additional acquisition of functional MR. In addition, our proposed SPAM-assisted presurgical mapping method would be beneficial when there was a lack of anatomical information.

\section{Conclusion}

Anatomical information about brain gliomas could be successfully extracted using SPAM. This proposed method would be an option for presurgical mapping, that could help to prevent induction of neurological deficits during surgery without the need for additional functional mapping studies.

\section{Acknowledgements}

This work was supported by the Korea Science and Engineering Foundation (KOSEF) (No. 2015001667 (PI: Joo Hyun Kang) and No. 1711026888 (PI: Kook-Hyun Yu)). 


\section{References}

[1] Zukotynski, K., Fahey, F., Kocak, M., et al. (2014) 18F-FDG PET and MR Imaging Associations across a Spectrum of Pediatric Brain Tumors: A Report from the Pediatric Brain Tumor Consortium. Journal of Nuclear Medicine, 55, 1473-1480.

[2] Schwenzer, N.F., Stegger, L., Bisdas, S., et al. (2012) Simultaneous PET/MR Imaging in a Human Brain PET/MR System in 50 Patients-Current State of Image Quality. European Journal of Radiology, 81, 3472-3478.

[3] Jeong, S.Y. and Lim, S.M. (2012) Comparison of 3'-Deoxy-3'-[18F]fluorothymidine PET and O-(2-[18F]Fluoroethyl)L-tyrosine PET in patients with newly diagnosed glioma. Nuclear Medicine and Biology, 39, 977-981.

[4] Gempt, J., Bette, S., Ryang, Y.M., et al. (2015) ${ }^{18}$ F-Fluoro-ethyl-tyrosine Positron Emission Tomography for Grading and Estimation of Prognosis in Patients with Intracranial Gliomas. European Journal of Radiology, 84, 955-962. http://dx.doi.org/10.1016/j.ejrad.2015.01.022

[5] Peck, K.K., Bradbury, M., Petrovich, N., et al. (2009) Presurgical Evaluation of Language Using Functional Magnetic Resonance Imaging in Brain Tumor Patients with Previous Surgery. Neurosurgery, 64, 644-652; discussion 652-643.

[6] Nadkarni, T.N., Andreoli, M.J., Nair, V.A., et al. (2015) Usage of fMRI for Pre-Surgical Planning in Brain Tumor and Vascular Lesion Patients: Task and Statistical Threshold Effects on Language Lateralization. NeuroImage: Clinical, 7, 415-423. http://dx.doi.org/10.1016/j.nicl.2014.12.014

[7] Partovi, S., Jacobi, B., Rapps, N., et al. (2012) Clinical Standardized fMRI Reveals Altered Language Lateralization in Patients with Brain Tumor. AJNR American Journal of Neuroradiology, 33, 2151-2157.

[8] Picht, T., Krieg, S.M., Sollmann, N., et al. (2013) A Comparison of Language Mapping by Preoperative Navigated Transcranial Magnetic Stimulation and Direct Cortical Stimulation during Awake Surgery. Neurosurgery, 72, 808-819.

[9] Kang, K.W., Lee, D.S., Cho, J.H., et al. (2001) Quantification of F-18 FDG PET Images in Temporal Lobe Epilepsy Patients Using Probabilistic Brain Atlas. Neuroimage, 14, 1-6. http://dx.doi.org/10.1006/nimg.2001.0783

[10] Choi, J.Y., Lee, K.H., Na, D.L., et al. (2007) Subcortical Aphasia after Striatocapsular Infarction: Quantitative Analysis of Brain Perfusion SPECT Using Statistical Parametric Mapping and a Statistical Probabilistic Anatomic Map. Journal of Nuclear Medicine, 48, 194-200.

[11] Lee, S.K., Lee, D.S., Yeo, J.S., et al. (2002) FDG-PET Images Quantified by Probabilistic Atlas of Brain and Surgical Prognosis of Temporal Lobe Epilepsy. Epilepsia, 43, 1032-1038. http://dx.doi.org/10.1046/j.1528-1157.2002.29701.x

[12] Lee, D.S., Lee, J.S., Kang, K.W., et al. (2001) Disparity of Perfusion and Glucose Metabolism of Epileptogenic Zones in Temporal Lobe Epilepsy Demonstrated by SPM/SPAM Analysis on $15 \mathrm{O}$ Water PET, [18F]FDG-PET, and [99mTc]HMPAO SPECT. Epilepsia, 42, 1515-1522. http://dx.doi.org/10.1046/j.1528-1157.2001.21801.x

[13] Lee, J.S., Lee, D.S., Kim, J., et al. (2005) Development of Korean Standard Brain Templates. Journal of Korean Medical Science, 20, 483-488. http://dx.doi.org/10.3346/jkms.2005.20.3.483

[14] Kim, J.S., Lee, J.S., Park, M.H., et al. (2010) Feasibility of Template-Guided Attenuation Correction in Cat Brain PET Imaging. Molecular Imaging and Biology, 12, 250-258.

[15] Kim, J.S., Kim, K.M., Kim, B.I., et al. (2008) Comparison of Registration Methods for the Clinical Applications of Multimodality Brain Glioma Images. 2008 Korean Society of Radiation Bioscience.

[16] Stevens, M.T., Clarke, D.B., Stroink, G., Beyea, S.D. and D’Arcy, R.C. (2015) Improving fMRI Reliability in Presurgical Mapping for Brain Tumours. Journal of Neurology, Neurosurgery \& Psychiatry. http://dx.doi.org/10.1136/jnnp-2015-310307

[17] Leung, H., Zhu, C.X., Chan, D.T., et al. (2015) Ictal High-Frequency Oscillations and Hyperexcitability in Refractory Epilepsy. Clinical Neurophysiology, in Press. http://dx.doi.org/10.1016/j.clinph.2015.01.009

[18] Guedj, E., Bonini, F., Gavaret, M., et al. (2015) ${ }^{18}$ FDG-PET in Different Subtypes of Temporal Lobe Epilepsy: SEEG Validation and Predictive Value. Epilepsia, 56, 414-421.

[19] Hong, S.J., Kim, H., Schrader, D., Bernasconi, N., Bernhardt, B.C. and Bernasconi, A. (2014) Automated Detection of Cortical Dysplasia Type II in MRI-Negative Epilepsy. Neurology, 83, 48-55. 